Article

\title{
Achieving a 3D Thermally Conductive while Electrically Insulating Network in Polybenzoxazine with a Novel Hybrid Filler Composed of Boron Nitride and Carbon Nanotubes
}

\author{
Yi Wang ${ }^{1}$, Wei Wu ${ }^{1, *}$, Dietmar Drummer ${ }^{2}$, Chao Liu ${ }^{1}$, Florian Tomiak ${ }^{2}$, Kevin Schneider ${ }^{2}$ and \\ Zhengqiang Huang ${ }^{1}$ \\ 1 Sino-German Joint Research Centre of Advanced Materials, School of Materials Science and Engineering, \\ East China University of Science and Technology, Shanghai 200237, China; \\ Y20150057@ecust.mail.edu.cn (Y.W.); Y30170440@ecust.mail.edu.cn (C.L.); \\ Y30180265@ecust.mail.edu.cn (Z.H.) \\ 2 Institute of Polymer Technology, Friedrich Alexander University Erlangen-Nuremberg, \\ 91058 Erlangen, Germany; drummer@lkt.uni-erlangen.de (D.D.); tomiak@lkt.uni-erlangen.de (F.T.); \\ schneider@lkt.uni-erlangen.de (K.S.) \\ * Correspondence: wuwei@ecust.edu.cn; Tel.: +86-64250850
}

Received: 19 August 2020; Accepted: 28 September 2020; Published: 13 October 2020

\begin{abstract}
To solve the problem of excessive heat accumulation in the electronic packaging field, a novel series of hybrid filler (BN@CNT) with a hierarchical "line-plane" structure was assembled via a condensation reaction between functional boron nitride(f-BN) and acid treated carbon nanotubes (a-CNTs). The reactions with different mass ratios of BN and CNTs and the effect of the obtained hybrid filler on the composites' thermal conductivity were studied. According to the results, BN@15CNT exhibited better effects on promoting thermal conductivity of polybenzoxazine(PBz) composites which were prepared via ball milling and hot compression. The thermally conductive coefficient value of $\mathrm{PBz}$ composites, which were loaded with $25 \mathrm{wt} \%$ of BN@15CNT hybrid fillers, reached $0.794 \mathrm{~W} \cdot \mathrm{m}^{-1} \cdot \mathrm{K}^{-1}$. The coefficient value was improved to $0.865 \mathrm{~W} \cdot \mathrm{m}^{-1} \cdot \mathrm{K}^{-1}$ with $15 \mathrm{wt} \%$ of BN@15CNT and $10 \mathrm{wt} \%$ of BN. Although CNTs were adopted, the PBz composites maintained insulation. Dielectric properties and thermal stability of the composites were also studied. In addition, different thermal conduction models were used to manifest the mechanism of BN@CNT hybrid fillers in enhancing thermal conductivity of PBz composites.
\end{abstract}

Keywords: fillers; functionalization of polymers; networks; nanocomposites

\section{Introduction}

Electronic and telecommunication industries have constantly developed for over half a century, corresponding to Moore's Law [1]. However, this development seems to have slowed down in recent years. If we evaluate the progress of computers with the computing power per joule for the last 50 years, it can be observed that the ability of computers doubled every 2.5 years, which is a little slower than that predicted by Moore. The main reason is the loop of heat accumulation-increasing operating temperature- extensive heat generation. Part of the input energy is transferred into heat during operation, which accumulates in the electronic components and causes increasing working temperature. Then more energy is transferred into heat owing to the rising temperature. The growing packing density accelerates this process because of the increasing power density [2]. A report revealed that every 2 degrees increase in temperature would lead to a $10 \%$ degradation in the performance of 
electronic devices [3].Therefore, developing efficient heat-dissipating materials is a critical task for preserving reliability [4].

Polymers including polyamide (PA6), epoxy, etc. [5], have been used as packaging materials because of their low density, low cost and high processability [6]. A newly developed thermosetting phenolic resin polybenzoxazine ( $\mathrm{PBz})$ [7], is a promising material for the usage in microelectronics and packaging industries [8]. Polybenzoxazine, which is synthesized through Mannich condensation from amine, formaldehyde and phenol [9], possesses excellent properties such as low moisture absorption, stable dielectric constant and flame retardance [10]. In addition, PBz has splendid mechanical properties and thermal properties [11].

However, their application has been limited because of the relatively low intrinsic thermally conductive coefficient $(\lambda)$ [12] $\left(0.1-0.3 \mathrm{~W} \cdot \mathrm{m}^{-1} \cdot \mathrm{K}^{-1}\right)$. The most widely used approach is to simply blend polymers with fillers with high $\lambda$ values, including carbon-based materials, metal, and ceramic $[13,14]$. In general, with the same content of filler, $\lambda$ values of the composites filled with carbon-based fillers, such as graphene or carbon nanotubes (CNTs) [15], are more likely to be higher than those of the composites filled with ceramic fillers such as boron nitride. CNTs $\left(\sim 1300 \mathrm{~W} \cdot \mathrm{m}^{-1} \cdot \mathrm{K}^{-1}\right)$ [16] have been frequently used in fabrication of thermally conductive polymer composites, but the reported experimental results for the $\lambda$ values of those composites are far less than those estimated from the rule of the simple blending model and the intrinsic thermal conductivity of CNTs [17]. Theoretically speaking, it is believed that the phonon scattering phenomenon in composite materials, achieved by acoustic mismatch, is the main reason impeding the further improvement of the thermal conductivity of those composites [18]. In addition, research revealed that when dispersed in polymers, CNTs are inclined to aggregate into bundles or ropes because of the strong intrinsic Van der Waals forces, which would also cause the diversity in theoretical and experimental results [19].

However, for applications in the electronics packaging field, polymer composites must be electrically insulating. Therefore, ceramic fillers have been widely employed, such as AlN [20], BN [21], $\mathrm{SiC}$ [22], etc. Among them, BN seems to be the most promising filler, owing to its high $\lambda$ value $\left(33 \mathrm{~W} \cdot \mathrm{m}^{-1} \cdot \mathrm{K}^{-1} \sim 600 \mathrm{~W} \cdot \mathrm{m}^{-1} \cdot \mathrm{K}^{-1}\right)$ and relatively low dielectric constant (approximately four), compared with those of other ceramic fillers [23]. Lei, Y. [24] conducted research on the thermal conductivity of h-BN filled epoxy/cyanate resin composites. According to Agari's model [25], the obtained data revealed that it is hard for h-BN to form effective conductivity channels. Therefore, fillers with large aspect ratios as well as high thermal conductivities [26], such as CNTs, were employed to assist the construction of the channels. Yang Xue [27] constructed a thermally conductive network with BN (30 phr) and CNTs ( $0.25 \mathrm{vol} \%$ ) in silicone rubber, whose $\lambda$ value was improved to $0.279 \mathrm{~W} \cdot \mathrm{m}^{-1} \cdot \mathrm{K}^{-1}$, $25 \%$ higher than that without CNTs. This research revealed that CNTs contribute to the construction of thermally conductive channels. However, with simple blending, the agglomeration of CNTs can hardly be avoided, which means that only part of the CNTs contribute to connecting the BN platelets [28]. Moreover, because of CNTs' interfacial boundaries and defect scattering, the agglomeration of CNTs undermines their ability to enhance thermal conductivity of the matrix [15].

Recently, controlling the distribution of filler to form robust heat transfer pathway has been proven to be an effective strategy to improve thermal conductivity of polymer-based composites [29]. For instance, Yimin Yao, et al. [30] adopted the combination of the ice-template and infiltrating method to construct BN platelets into a three-dimensional network, which were stacked on reduced graphene oxide(rGO). The reported 3D rGO-BN/epoxy composites exhibited an remarkable $\lambda$ value of $5.05 \mathrm{~W} \cdot \mathrm{m}^{-1} \cdot \mathrm{K}^{-1}$ at a filler loading of $13.16 \mathrm{vol} . \%$. Yongqiang Guo et al. [2] constructed a new kind of fully carbon-based filler (f-MWCNT-g-rGO) with graphene oxide (GO) and multi-walled carbon nanotubes(MWCNTs). The thermally conductive polyimide nanocomposites (f-MWCNT-g-rGO/PI) were then prepared via a successive method of in situ polymerization, electrospinning and hot pressing. The fabricated fillers have a hierarchical structure, resulting in the outstanding thermal conductivity coefficient of $1.60 \mathrm{~W} \cdot \mathrm{m}^{-1} \cdot \mathrm{K}^{-1}$ at a relatively low loading of fillers (10 $\left.\mathrm{wt} \%\right)$. 
In this work, CNTs and h-BN were assembled together at different mass ratios via a condensation reaction to prepare a hybrid filler (BN@CNT) with a hierarchical "line-plane" structure, which was designed to effectively form a thermally conductive network throughout the polymer matrix. The BN@CNT/PBz composites were prepared via powder blending and then hot compression. The effects of structures of the as prepared hybrid fillers on thermal conductivity composites were investigated. In addition, $\mathrm{PBz}$ composites with high thermal conductivity were fabricated when BN and BN@CNT were simultaneously employed as fillers. Furthermore, to fully understand the effect of BN@CNT hybrid filler on enhancing thermal conductivity of PBz composites, the experimental data were fitted with a simple effective medium approximation (EMA) model and Foygel's model to calculate thermal boundary resistance $\left(R_{B}\right)$ and contact thermal resistance $\left(R_{c}\right)$.

\section{Materials and Methods}

\subsection{Materials}

Benzoxazine resin (BOZ) was supplied by Ruiyi Chemical Technology Co., Ltd.(Shanghai, China), $\left(\right.$ AIBZ682, density $\left.=1.20 \mathrm{~g} \cdot \mathrm{cm}^{-3}\right)$. Hexagonal boron nitride $(50 \mu \mathrm{m})(\mathrm{h}-\mathrm{BN})$ was purchased from ZiboJonye Ceramic Technologies Co., Ltd. (Shandong, China).; carbon nanotubes (CNTs), with the average diameter of 30 50 $\mathrm{nm}$ and length of $10 \mu \mathrm{m}$, were purchased from Shenzhen Zhongke Nano New Material Co. Ltd. (Shenzhen, China), Sodium hydroxide was supplied by Shanghai Aladdin Biochemical Technology Co., Ltd. (Shanghai, China); 3-triethoxysilylpropylamine (>98\%) (APTES) was purchased from Shanghai Yuanye Biotechnology Co., Ltd.; N,N'-diisopropylcarbodiimide (DIC), sulfuric acid (98\%), nitric acid (68\%), absolute ethanol and tetrahydrofuran (THF) were all purchased from Shanghai Titan Scientific Co., Ltd. (Shanghai, China).

\subsection{Specimen Preparation}

a-CNT Preparation. To obtain the carboxyl grafted CNTs, $1 \mathrm{~g}$ CNTs was added into $30 \mathrm{~mL}$ sulfuric acid (98\%) and $10 \mathrm{~mL}$ nitric acid $(68 \%)$ and ultrasonicated for $4 \mathrm{~h}$ at $60{ }^{\circ} \mathrm{C}$ [31]. The slurry was centrifuged for $1.5 \mathrm{~h}$, and then washed with DI water until the $\mathrm{pH}$ of the mixture was 6.5. After being ultrasonicated for $1 \mathrm{~h}$, the slurry was placed in afreezer at $-18{ }^{\circ} \mathrm{C}$ to be frozen for $12 \mathrm{~h}$. After freeze-drying for $48 \mathrm{~h}$, the acid-treated CNT (a-CNT) was obtained.

f-BN Preparation. Firstly, $20 \mathrm{~g}$ h-BN was dispersed in $200 \mathrm{~mL} 5 \mathrm{M}$ aqueous sodium hydroxide solution and stirred magnetically for $24 \mathrm{~h}$ at $120^{\circ} \mathrm{C}$. The mixture was alternately filtered and washed three times with DI water to remove the redundant sodium hydroxide. After drying at $120^{\circ} \mathrm{C}$ for $24 \mathrm{~h}, \mathrm{BN}-\mathrm{OH}$ was obtained. Secondly, APTES ( $3 \%$ of the weight of obtained BN-OH) was dissolved in a $95 \mathrm{wt} \%$ aqueous ethanol solution, the $\mathrm{pH}$ of which was adjusted to 5 . Then $\mathrm{BN}-\mathrm{OH}$ was added into the solution and stirred magnetically for $6 \mathrm{~h}$ at $80^{\circ} \mathrm{C}$. The slurry was then vacuum dried at $120^{\circ} \mathrm{C}$ for $24 \mathrm{~h}$. Finally, the obtained powder was washed three times with ethanol and DI water to remove unreacted APTES. After drying at $120^{\circ} \mathrm{C}$ for $24 \mathrm{~h}$, light yellow particles were obtained which were coded as f-BN.

BN@CNT Preparation. a-CNT, f-BN and DIC were added into THF, followed by ultrasonic treatment for $2 \mathrm{~h}$ and stirred magnetically for $48 \mathrm{~h}$ at $65^{\circ} \mathrm{C}$. The mixture was washed with DI water through centrifugation and underwent further drying. The formula is list in Table 1 . The obtained particles were named as BN@xCNT $(x=5 / 10 / 15 / 20)$. The whole procedure is shown in Figure 1

Table 1. Formula of BN@xCNT.

\begin{tabular}{ccccc}
\hline & BN (g) & CNT (g) & THF (L) & DIC (g) \\
\hline BN@5CNT & 10 & 0.5 & 0.5 & 2 \\
BN@10CNT & 10 & 1 & 1 & 2 \\
BN@15CNT & 10 & 1.5 & 1.5 & 2 \\
BN@20CNT & 10 & 2 & 2 & 2 \\
\hline
\end{tabular}




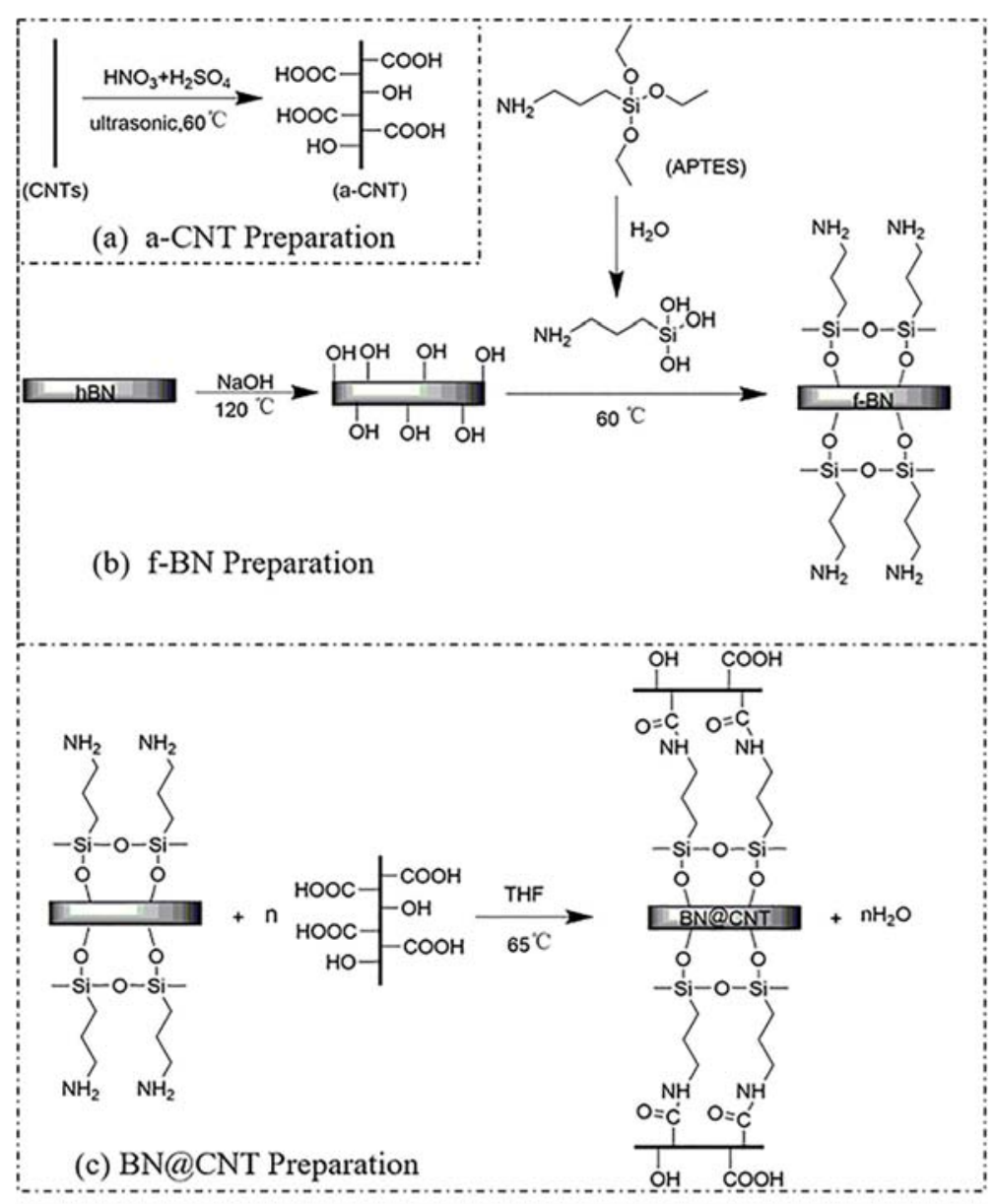

Figure 1. Illustration of the preparation procedures of BN@CNT hybrid filler.

Fabrication of thermally conductive BN@xCNT/PBz composites. As prepared BN@xCNT and $\mathrm{BOZ}$ were blended together through ball milling at $170 \mathrm{rpm}$ for $2 \mathrm{~h}$. Then the blended powder was filled into a certain mold and hot pressed under $12 \mathrm{MPa}$ following the heating strategy of $160{ }^{\circ} \mathrm{C} / 1 \mathrm{~h}$ $+180{ }^{\circ} \mathrm{C} / 1 \mathrm{~h}+200{ }^{\circ} \mathrm{C} / 1 \mathrm{~h}+220^{\circ} \mathrm{C} / 1 \mathrm{~h}$. The curing mechanism is shown in Figure 2. For comparison, $\mathrm{BN} / \mathrm{PBz}$ composites, $\mathrm{CNTs} / \mathrm{PBz}$ composites and $\mathrm{BN} / \mathrm{CNTs} / \mathrm{PBz}$ composites were prepared following the same strategy, in which BN/CNTs refersto simple blending of BN and CNTs at the same mass ratio as that of BN@15CNT.

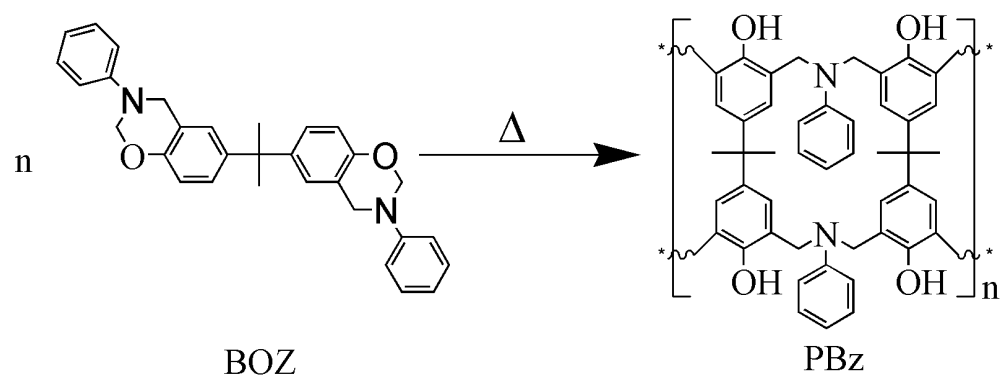

Figure 2. Curing mechanism of PBz.

\subsection{Characterizations}

Fourier transform infrared (FTIR) spectra was conducted on an IR spectrometer (IRAffinity-1, Kyoto, Japan) from $400 \mathrm{~cm}^{-1}$ to $4000 \mathrm{~cm}^{-1}$. 
A scanning electron microscope (SEM, S-3400, Hitachi Ltd., Japan) was used to observe the morphology of the hybrid filler particle and fabricated composites. Samples were fractured in liquid nitrogen.

Thermogravimetric analysis (TGA) of the specimens were carried out by a 2P-WRT (Shanpin Instrument Co., Shanghai, Ltd., Shanghai, China) over a range of temperature $\left(50-750{ }^{\circ} \mathrm{C}\right)$; all the filler samples were tested in air atmosphere at $10^{\circ} \mathrm{C} / \mathrm{min}$, while polymeric composites were tested in nitrogen atmosphere at $10{ }^{\circ} \mathrm{C} / \mathrm{min}$.

A broadband dielectric spectrometer (Novocontrol Technology Company, Germany) was used to measure the dielectric constant $(\varepsilon)$ and dielectric loss factor $(\tan \delta)$ values of composites. The testing frequency range was from $10^{-1}$ to $10^{7} \mathrm{~Hz}$. Samples were cylindrical shaped of $21.0 \mathrm{~mm}$ in diameter and $1 \mathrm{~mm}$ in thickness.

A high resistivity meter, model LK2679A was used to measure the volume resistivity (R) of composites at $250 \mathrm{~V}$. The corresponding dimension of specimen was $80 \mathrm{~mm} \times 80 \mathrm{~mm} \times 1 \mathrm{~mm}$.

A thermal conductivity instrument TC3000E (Xi'an Xiaxi Electronic Technology Co., Ltd., $\mathrm{Xi}^{\prime}$ an, China) was employed to measure the thermally conductive coefficient $(\lambda)$ of the samples. The corresponding dimensions of the specimens were $60 \mathrm{~mm} \times 40 \mathrm{~mm} \times 2 \mathrm{~mm}$.

\section{Results and Discussion}

\subsection{Characterization of BN@xCNT Hybrid Filler}

As is shown in Figure 3a, peaks at $2918 \mathrm{~cm}^{-1}$ and $1401 \mathrm{~cm}^{-1}$ corresponded to the CNT core [32]. The peak at $3416 \mathrm{~cm}^{-1}$ resulted from the stretching vibration of $-\mathrm{OH}$, and the stretching vibration of $-\mathrm{C}=\mathrm{O}-$ and $-\mathrm{C}-\mathrm{O}$ - appeared at $1617 \mathrm{~cm}^{-1}$ and $1107 \mathrm{~cm}^{-1}$, respectively. These peaks revealed that $-\mathrm{OH}$ and $-\mathrm{COOH}$ were introduced to $\mathrm{CNTs}$ after acid treatment. The characteristic absorption peaks of h-BN platelets can be seen in Figure $3 \mathrm{~b}$ at $1374 \mathrm{~cm}^{-1}$ and $819 \mathrm{~cm}^{-1}$, indicating the B-N stretching vibration and the B-N-B out-of-plane bending, respectively [33]. Peaks at $2968 \mathrm{~cm}^{-1}$ (stretching vibration of $\mathrm{C}-\mathrm{H}$ ) and $635 \mathrm{~cm}^{-1}$ (out-of-plane bending vibration of $\mathrm{N}-\mathrm{H}$ ) indicated the existence of APTES. In Figure $3 c$, the peak at $1614 \mathrm{~cm}^{-1}$ resulting from stretching vibration of $C=O$ revealed the formation of amide bonds, which connected f-BN and a-CNTs.

Morphologies of BN@xCNT with different weight ratio of CNTs are shown in Figure 4. In Figure 4a the surface of BN flakes is smooth. With the increasing content of CNTs, the distribution of CNTs varied. In Figure $4 b, c$, it can be observed that most a-CNTs dispersed on the surface of h-BN with no sign of large agglomerate. With further increasing content of a-CNTs, it can be observed from Figure $4 \mathrm{~d}$ that some of a-CNTs stacked at the edge of $\mathrm{f}-\mathrm{BN}$ and connected several h-BN particles together. As seen in Figure 4e, a-CNTs covered the whole surface of $\mathrm{f}-\mathrm{BN}$ when the content of CNTs kept increasing. It can be estimated that the differences in location of a-CNTs would lead to the differences in thermal conductivity of BN@xCNT/PBz composites. 


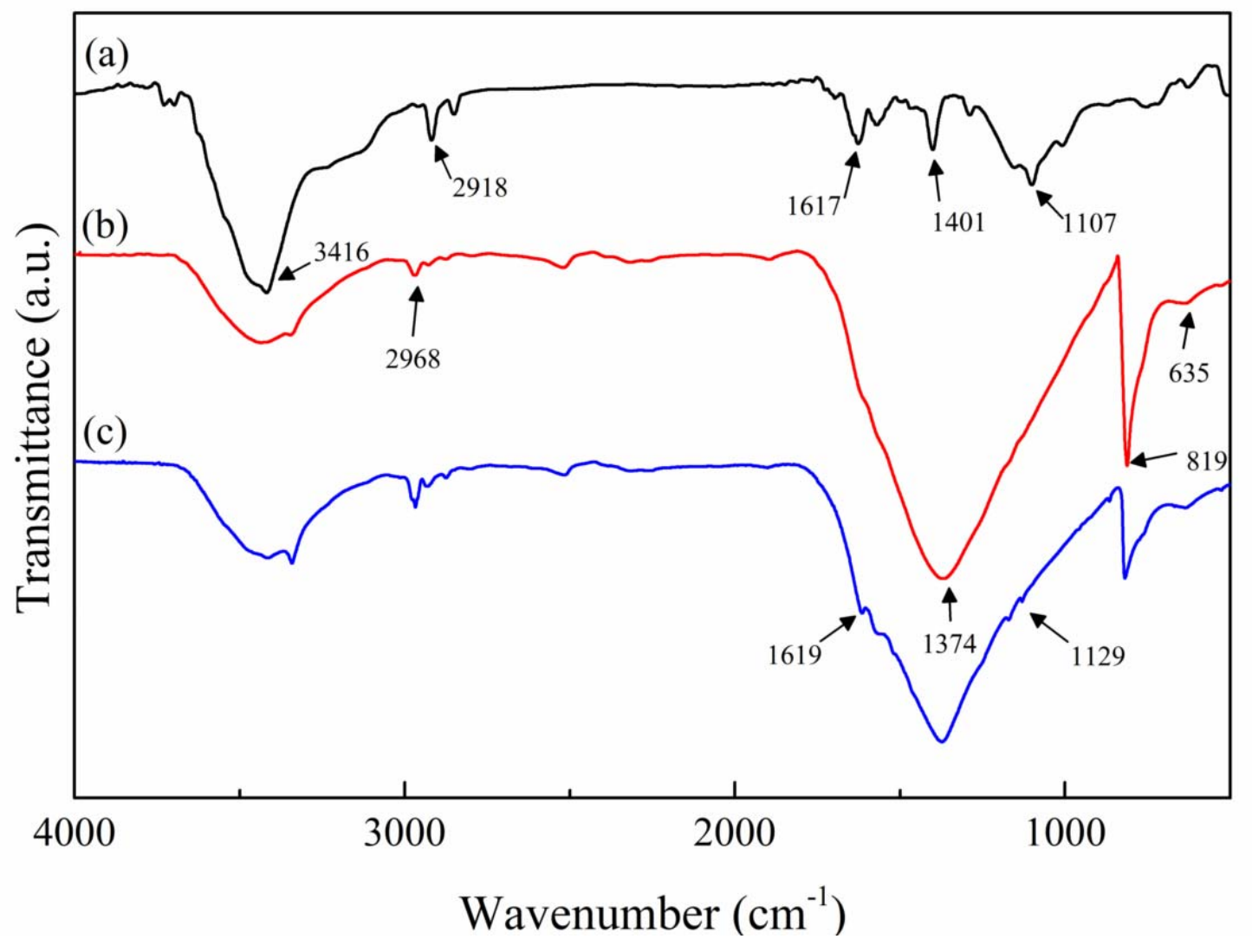

Figure 3. FT-IR spectra of (a) a-CNT; (b) f-BN; and (c) BN@15CNT.
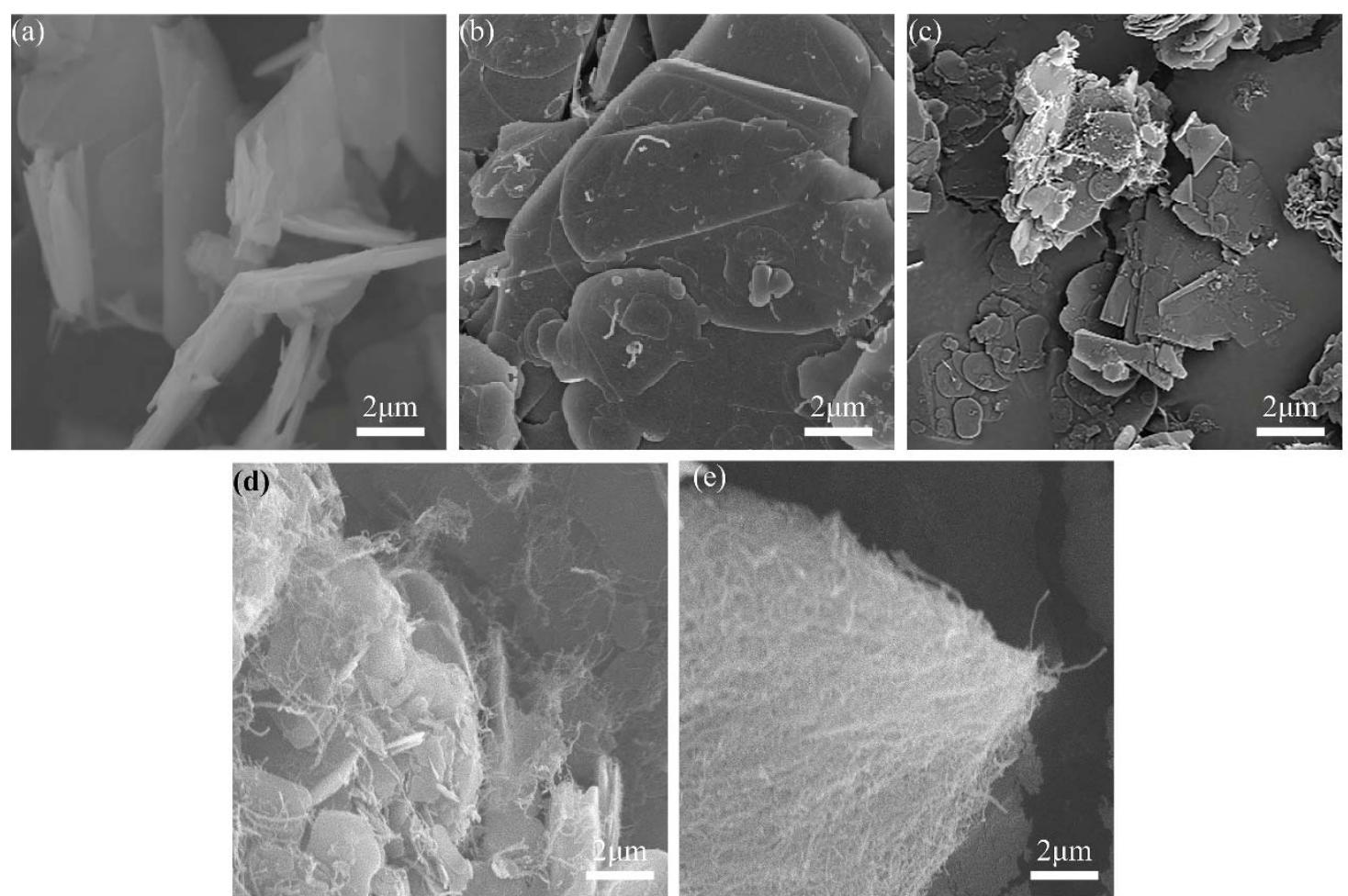

Figure 4. SEM image of BN@xCNT hybrid fillers of (a) pristine h-BN;(b) BN@5CNT; (c) BN@10CNT; (d) BN@15CNT; and (e) BN@20CNT. 
To quantitatively analyze the content of CNT in prepared BN@xCNT, TGA under air atmosphere was conducted on pristine h-BN, f-BN, BN@xCNT and a-CNTs. The corresponding TGA curves are presented in Figure 5. The pristine h-BN platelet with high thermal stability did not show obvious weight loss. f-BN showed slight weight loss resulting from the decomposition of APTES [34]. Furthermore, a-CNT kept losing weight from $100^{\circ} \mathrm{C}$, resulting from the decomposition of -OH groups, $-\mathrm{COOH}$ groups and defect points formed during acid treatment. The obvious weight loss for a-CNTs over $550{ }^{\circ} \mathrm{C}$ was attributed to the decomposition of its core structure. BN@xCNT decomposed in a similar pattern as did a-CNT. According to following equations, the content of CNTs in prepared $\mathrm{BN} @ \mathrm{xCNT}$ and the conversion rate of the reaction can be calculated.

$$
\begin{gathered}
\Phi=\left(l_{1}-l_{2}\right) /\left(l_{3}-l_{2}\right) \\
\alpha=\Phi / \Phi_{0}
\end{gathered}
$$

in which $1_{1}, 1_{2}, 1_{3}$ are the weight loss percentage of $\mathrm{BN} @ \mathrm{xCNT}$, f-BN and CNT, respectively; $\Phi_{0}$ and $\Phi$ stand for the content of a-CNTs before and after reaction; $\alpha$ means the conversion of the reaction. The data used and obtained results are listed in Table 2. It can be inferred from the $\alpha$ value that with the increasing of the a-CNTs and h-BN ratio, more a-CNTs were grafted onto f-BN, and a-CNTs were excessive when the ratio of CNTs and f-BN reached 1:5. Therefore, the $\alpha$ value of BN@20CNT dropped dramatically.

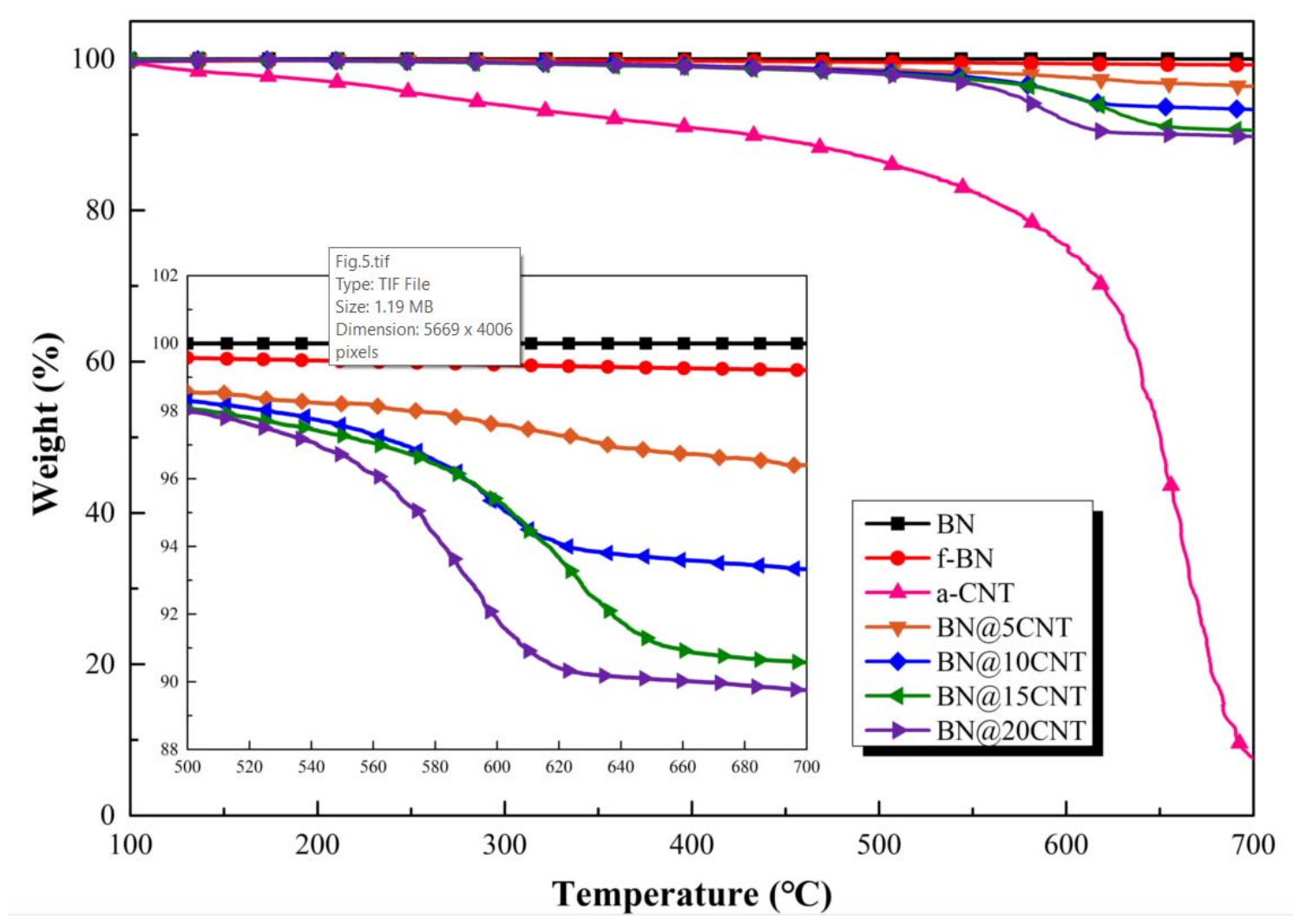

Figure 5. TGA curves of h-BN, f-BN, BN@xCNT and a-CNT. 
Table 2. Calculated results of content of CNTs and corresponding data used.

\begin{tabular}{cccc}
\hline & Weight Loss $(\mathbf{\%})$ & Content of CNTs $\mathbf{( \% )}$ & $\boldsymbol{\alpha} \mathbf{( \% )}$ \\
\hline BN@5CNT & 3.593 & 3.052 & 64.10 \\
BN@10CNT & 6.672 & 6.417 & 70.58 \\
BN@15CNT & 9.429 & 9.430 & 72.30 \\
BN@20CNT & 10.248 & 10.325 & 61.95 \\
\hline
\end{tabular}

\subsection{Effect of BN@xCNT Hybrid Fillers' Structure on the Thermal Conductivity of PBz Composites}

To fully determine the relationship between the content of CNTs in BN@xCNT hybrid fillers and their effect on thermal conductivity of corresponding PBz composites, filler loading over and below the percolation threshold were both investigated, since the pattern of thermal conductivity differs. According to former research, the percolation threshold in a similar system was about $20 \mathrm{wt} \%$ [35]. Therefore, $15 \mathrm{wt} \%$ and $25 \mathrm{wt} \%$ were chosen as the filler loading in this part. The corresponding $\lambda$ values are shown in Figure 6.

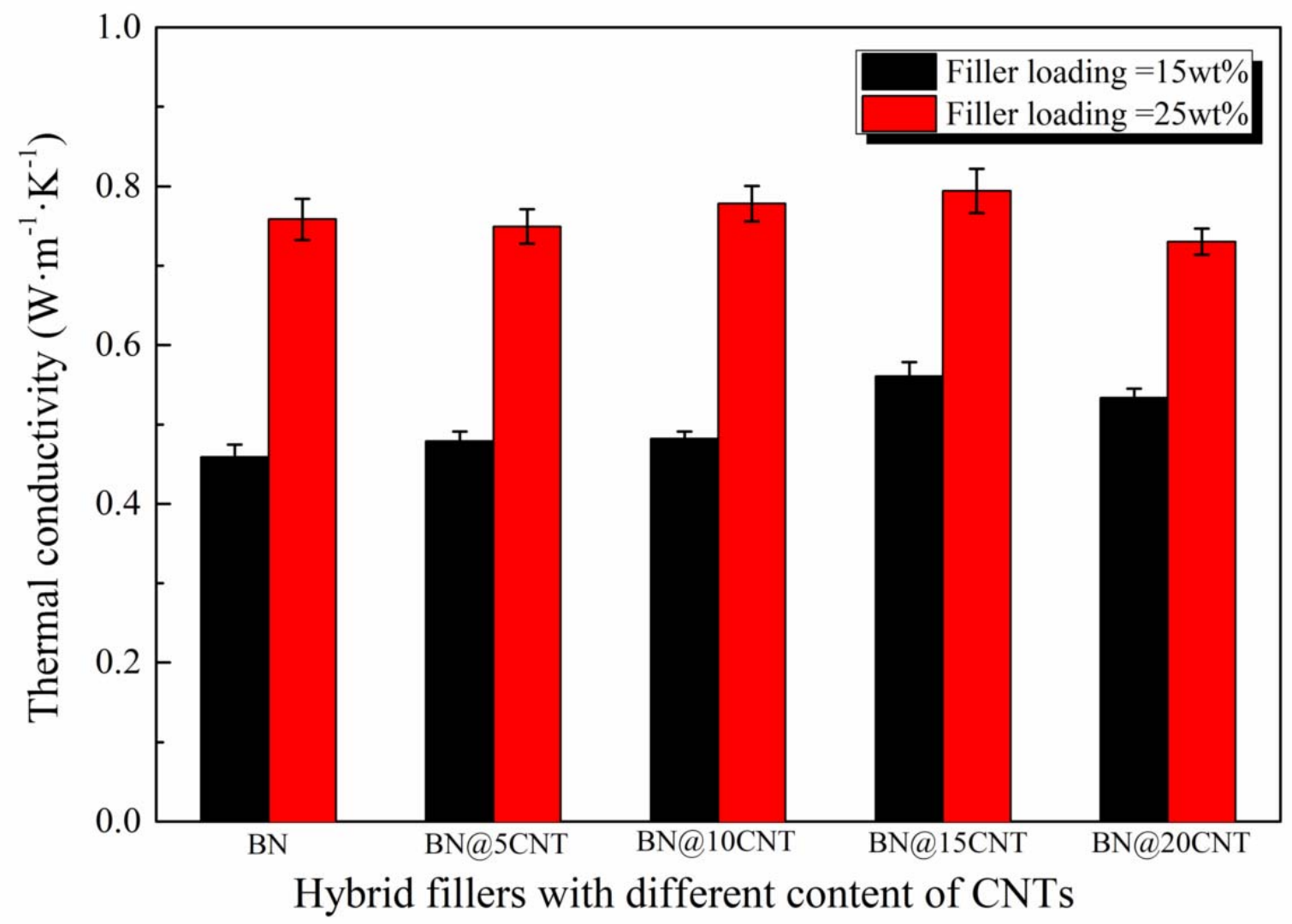

Figure 6. Thermal conductivity of PBz composites with $15 w t \%$ and $25 w t \%$ BN@xCNT hybrid fillers.

It can be observed that PBz composites filled with different BN@xCNT hybrid fillers performed various $\lambda$ values at the same filler loading. Such a discrepancy was more obvious at lower filler loading. Among all the hybrid fillers, BN@15CNT showed the best ability to improving thermal conductivity of $\mathrm{PBz}$ composites than other fillers, both at $15 \mathrm{wt} \%$ and $25 \mathrm{wt} \%$ loading amounts. The corresponding $\lambda$ values of $\mathrm{PBz}$ composites were improved from $0.459 \mathrm{~W} \cdot \mathrm{m}^{-1} \cdot \mathrm{K}^{-1}$ and $0.762 \mathrm{~W} \cdot \mathrm{m}^{-1} \cdot \mathrm{K}^{-1}$ to $0.564 \mathrm{~W} \cdot \mathrm{m}^{-1} \cdot \mathrm{K}^{-1}$ and $0.794 \mathrm{~W} \cdot \mathrm{m}^{-1} \cdot \mathrm{K}^{-1}$, respectively, comparing with pristine BN. It can be inferred that the differences in $\lambda$ values were caused by the combined action between the structure of hybrid fillers and filler loading. When the content of filler was under the percolation threshold, the BN platelets were not connected with each other. Therefore, the construction of a thermally conductive network 
depended on the CNTs to connect adjacent BN platelets. When the filler loading was over the percolation threshold, BN platelets could form a thermally conductive network themselves, which means CNTs contributed less to the formation of the network. This explained why the discrepancy in $\lambda$ values at $15 \mathrm{wt} \%$ were more obvious than that at $25 \mathrm{wt} \%$. At the same filler loading, the different structure of hybrid fillers resulting from various CNT contents dominated the enhancement in $\lambda$ values of the $\mathrm{PBz}$ composites. According to the SEM images of BN@5CNT and BN@10CNT hybrid fillers, the a-CNTs distributed on the surface of BN platelets so that they could hardly link the adjacent BN platelets, as shown in Figure 7a. Therefore, the corresponding $\lambda$ values were almost similar to those of $\mathrm{BN} / \mathrm{PBz}$ composites. When the a-CNT started to distribute on the edge of BN platelets, they could act as "bridges" to connect BN, which is shown in Figure 7b. In this way, the BN@15CNT hybrid filler exhibited the best ability to enhance the thermal conductivity of the $\mathrm{PBz}$ composites at both filler loading levels. It is worth noting that the $\lambda$ value of $\mathrm{BN} @ 20 \mathrm{CNT} / \mathrm{PBz}$ composites was higher than that of $\mathrm{BN} / \mathrm{PBz}$ composites at $15 \mathrm{wt} \%$ filler loading while the opposite was the case at $25 \mathrm{wt} \%$ filler loading. This could be attributed to competition between serious phonon scattering of a-CNT and formation of a thermally conductive network [36]. In the BN@20CNT hybrid filler, a-CNT covered BN platelets thoroughly, so that when the hybrid fillers connected with each other, it was the connection between a-CNT in most cases. Due to lattice mismatch and defects caused by acid treatment, the phonon scattering between the a-CNT connection was serious and led to high contact resistance [37]. At $15 \mathrm{wt} \%$ filler loading, a-CNT assisted the formation of the thermally conductive network which did not exist in BN/PBz composites via connecting adjacent BN platelets. Therefore, BN@20CNT/PBz composites exhibited better thermal conductivity than BN/PBz composites did. However, at $25 \mathrm{wt} \%$ filler loading, $\mathrm{BN}$ could form a thermally conductive network even without a-CNT, so that phonon scattering became the main reason influencing thermal conductivity of BN@20CNT/PBz composites.

(a)

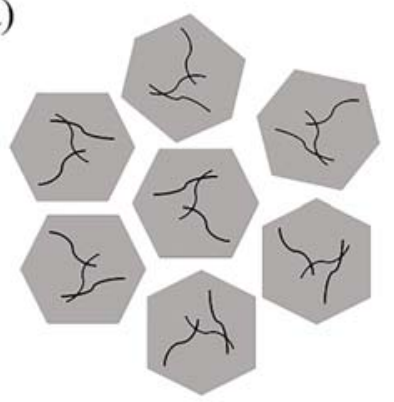

(b)

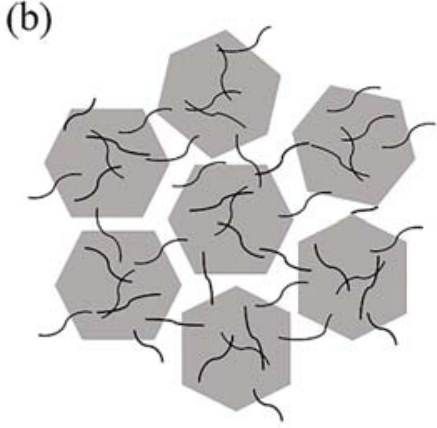

Figure 7. Schematic diagram of the effect of different BN@xCNT hybrid fillers on the heat transferring: (a) BN@5CNT; (b) BN@15CNT.

The following research focuses on the effect of the BN@15CNT hybrid filler on properties of PBz composites resulting from the fact that BN@15CNT exhibited the best ability to enhance thermal conductivity of $\mathrm{PBz}$ composites at both filler loadings compared with other fillers.

\subsection{Effect of BN@15CNT on Thermal Conductivity of PBz Composites}

Figure 8a depicts the $\lambda$ values of BN@15CNT/PBz, BN/CNTs/PBz and BN/PBz composites. It can be observed that $\lambda$ values of composites with the BN@15CNT or BN/CNTs were higher than those of composites with pristine BN at all filler loading levels. When the content of filler was no more than $20 \mathrm{wt} \%$, the BN@15CNT hybrid filler exhibited a better effect on enhancing thermal conductivity of $\mathrm{PBz}$ composites than BN/CNTs did. However, when the filler loading reached $25 \mathrm{wt} \%$, the $\lambda$ value of $\mathrm{BN} / \mathrm{CNTs} / \mathrm{PBz}$ composite reached $0.844 \mathrm{~W} \cdot \mathrm{m}^{-1} \cdot \mathrm{K}^{-1}$, while that of BN@15CNT/PBz composites and $\mathrm{BN} / \mathrm{PBz}$ composites was $0.794 \mathrm{~W} \cdot \mathrm{m}^{-1} \cdot \mathrm{K}^{-1}$ and $0.765 \mathrm{~W} \cdot \mathrm{m}^{-1} \cdot \mathrm{K}^{-1}$, respectively. The corresponding enhancement reached $270 \%, 249 \%$ and $235 \%$, respectively. 
(a)

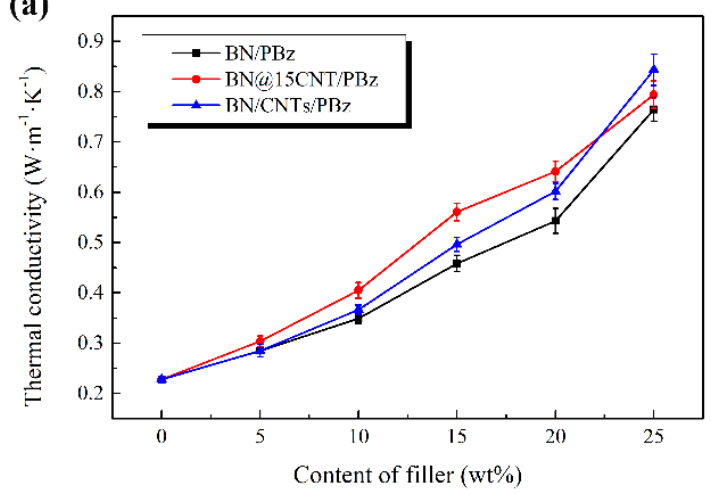

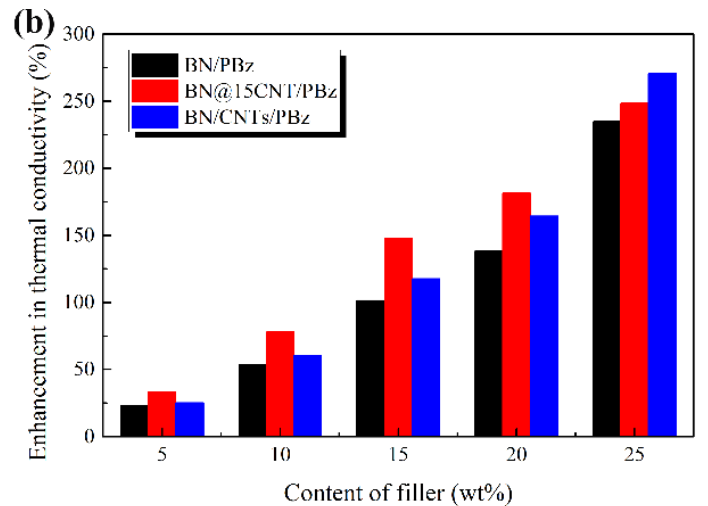

Figure 8. (a)Thermal conductivity of the PBz composites with different content of BN@15CNT and BN; (b) enhancement in thermal conductivity of BN@15CNT/PBz and BN/PBz composites.

It can be observed from the $\lambda$ values of $\mathrm{PBz}$ composites filled with these different fillers that the cooperation of $\mathrm{BN}$ and $\mathrm{CNTs}$, either in physical or chemical methods, led to synergetic effects on enhancing thermal conductivity of PBz composites. The main reason is that CNTs served as "bridges", establishing a connection between BN platelets, so as to improve the continuity of the thermal network. When the filler loading was less than $20 \mathrm{wt} \%, \mathrm{BN} @ 15 \mathrm{CNT} / \mathrm{PBz}$ composites showed better thermal conductivity than BN/CNTs/PBz composites did because the BN@15CNT hybrid filler ensured that CNTs distributed evenly among BN platelets so that the continuous thermally conductive network could be formed. However, in BN/CNTs/PBz composites, the distribution of CNTs would not be even because the simple blending of BN and CNTs could not avoid CNTs' agglomeration, which led to the fact that part of the BN platelets were connected with CNTs while part of them lacked connection with other fillers. When the content of filler reached $25 \mathrm{wt} \%$, BN platelets could form thermally conductive network themselves, so that the a-CNTs on their surface became less necessary while simple blended CNTs could form new channels because they were not constrained on the BN surface [38]. Moreover, the CNT connections between BN@15CNT hybrid fillers increased the contact resistance because of the phonon scattering caused by lattice mismatch when CNTs stacked together.

\subsection{Further Enhancement in Thermal Conductivity via the Synergistic Effect Between BN@15CNT and BN}

According to the former explanation, to achieve better enhancement in thermal conductivity, the interconnection between CNTs and BN should be maximized while that between CNTs should be avoided. To test this theory, BN was added into $15 \mathrm{wt} \% \mathrm{BN} @ 15 \mathrm{CNT} / \mathrm{PBz}$ composites. As it is shown in Figure 9, with the introduction of $\mathrm{BN}$, the $\lambda$ value of $\mathrm{PBz}$ composite increased more sharply than that of either BN@15CNT/PBz composites or BN/PBz composites. With $15 \mathrm{wt} \%$ of BN@15CNT and $10 \mathrm{wt} \%$ of $\mathrm{BN}$, the $\mathrm{PBz}$ composite's $\lambda$ value reached $0.865 \mathrm{~W} \cdot \mathrm{m}^{-1} \cdot \mathrm{K}^{-1}$. This obvious enhancement was ascribed to the fact that BN@15CNT offered sufficient "bridges" to connect other filler particles while BN dampened the chances of the overlapping of CNTs. When $15 \mathrm{wt} \%$ BN@15CNT hybrid filler was loaded, the thermally conductive network was about to be formed completely. Compared with further addition of BN@15CNT, the addition of BN would also help construct the network while the interconnection between CNTs would not increase, which conforms to the explanation proposed before. 


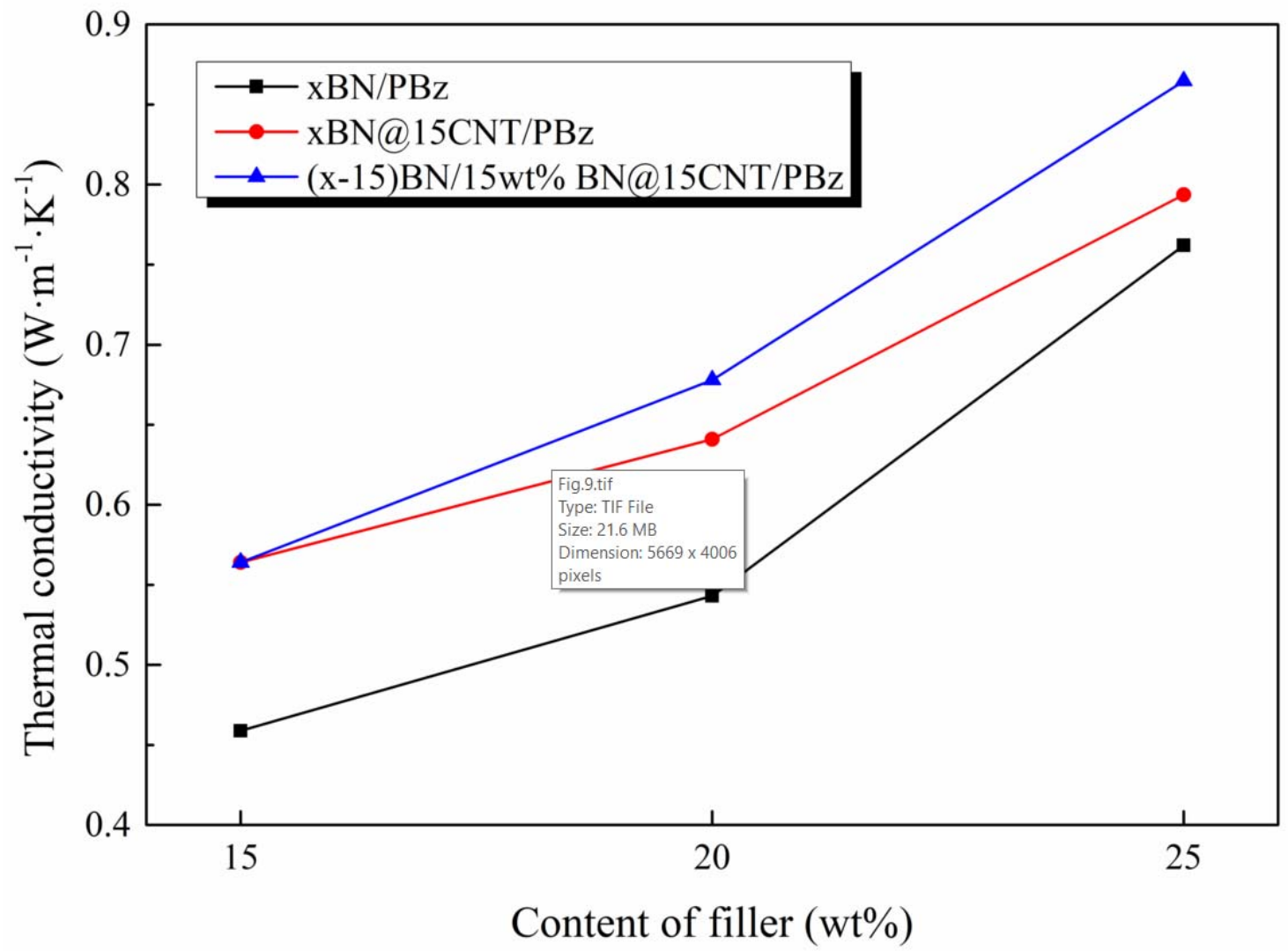

Figure 9. Thermal conductivity of the BN/BN@15CNT/PBz composites.

\subsection{Dielectric Properties of the BN@15CNT/PBz Composites}

The $\varepsilon$ and $\tan \delta$ values of the PBz composites with different content of BN@15CNT hybrid filler at different testing frequencies are presented in Figure 10. The $\varepsilon$ and $\tan \delta$ values both increased with increased filler loading at the same frequency. Except for the composite with $25 \mathrm{wt} \%$ filler content, $\varepsilon$ values of the composites showed little dependency on frequency. However, the tan $\delta$ values firstly increased, then decreased, the tendency of which became more obvious with higher filler loading. This tendency indicated the process of dielectric relaxation [39]. The corresponding $\varepsilon$ and $\tan \delta$ of the BN@15CNT/PBz composite with 25 wt\% BN@15CNT hybrid fillers was 1.60 and 0.028 at $1 \mathrm{MHz}$, respectively.

(a)

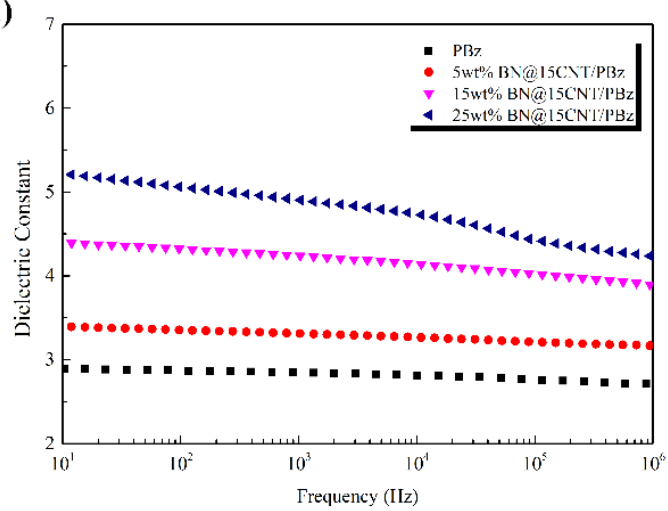

(b)

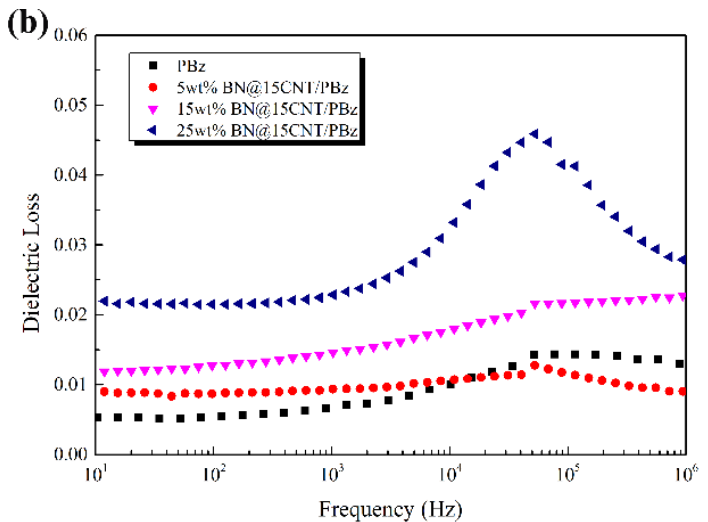

Figure 10. (a) $\varepsilon$ and (b) $\tan \delta$ values of PBz composites with different content of BN@15CNT at different testing frequency 
It can be inferred from the peak in tan $\delta$ values that pristine PBz performed viscoelastic relaxation because of dipolar relaxation, in which these dipoles were permanent dipoles present on the side chains of the polymer backbone [40] When the filler loading reached $25 \mathrm{wt} \%$, the relaxation behavior was extremely obvious, which resulted from the combination of viscoelastic relaxation and conductivity relaxation, which was due to translational diffusion of ions that caused conduction [41].

\subsection{Electrical Conductivity of BN@15CNT/PBz Composites}

For the application in electronic packaging, composite materials must be electrically insulated (volume resistivity $>10^{12} \Omega \cdot \mathrm{m}$ ). Figure 11 shows the volume resistivity of the composites as a function of filler content. A decrease in volume resistivity of BN@15CNT/PBz composites could be observed with the increases in filler content. This can be explained by the microstructure of the hybrid fillers. For the BN@15CNT hybrid fillers, CNTs were restricted on the surface of h-BN platelets. Hence the low content of BN@15CNT hybrid fillers would not achieve enough contact of CNTs. Although the contact of CNTs would increase with the higher content of hybrid filler, the network of CNTs were separated by h-BN platelets, and therefore the high insulation property could be maintained.

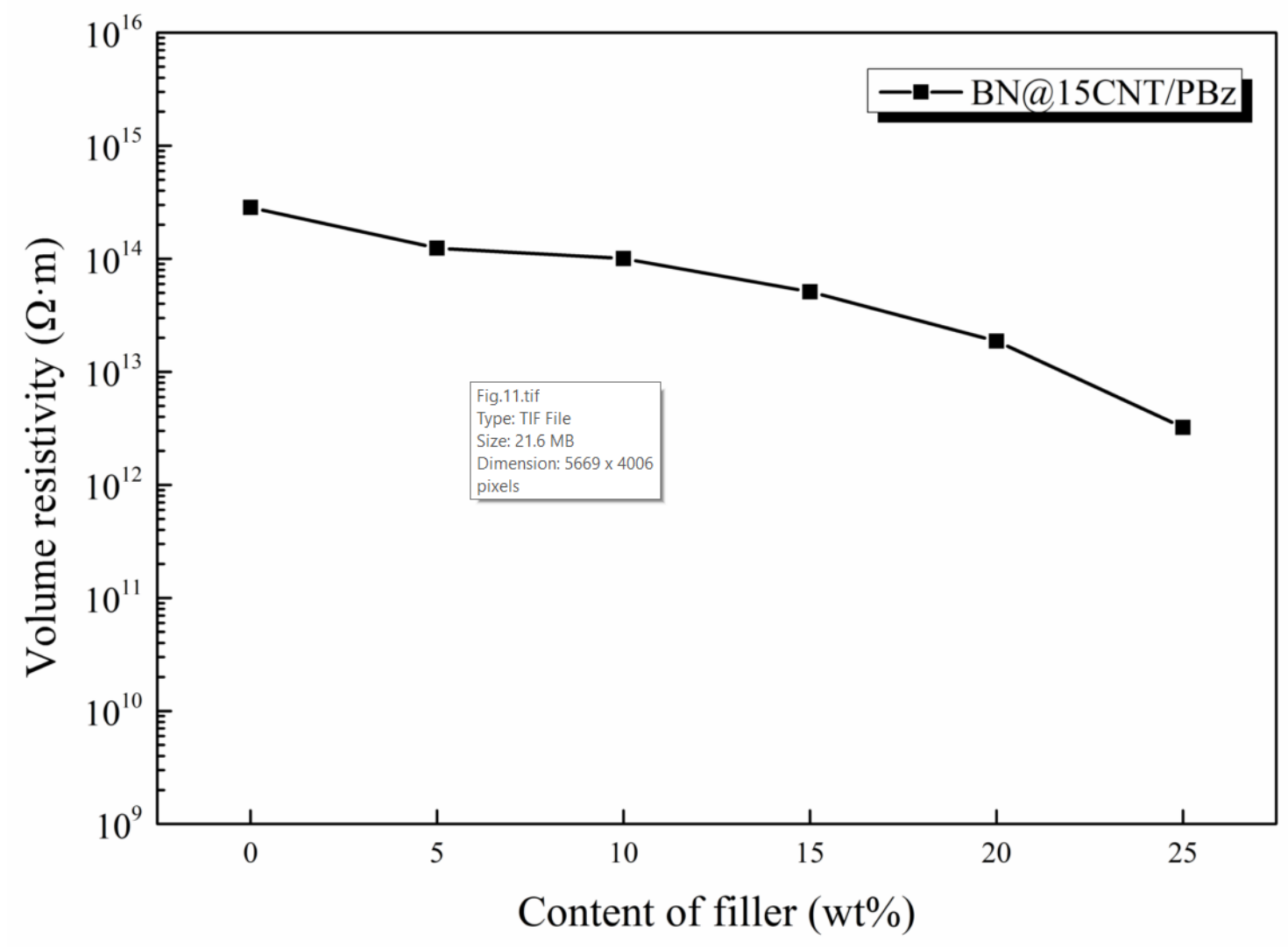

Figure 11. Volume resistivity of BN@15CNT/PBz composites as a function of filler content.

\subsection{Thermal Stability of BN@15CNT/PBz Composites}

To study the effect of BN@15CNT hybrid fillers on the thermal stability of PBz composites, BN@15CNT/PBz composites, BN/PBz composites and pristine PBz were characterized via TGA. The TG and DTG curves are shown in Figure 12 and corresponding characteristic thermal temperatures are listed in Table 3. It can be concluded that both BN@15CNT/PBz composites and BN/PBz composites exhibited a similar tendency, which is that the decomposition temperature of composites was reduced with the introduction of filler, although the higher content filler led to higher thermal decomposition temperature. The DTG curve of pristine PBz exhibited two peaks, revealing the two step decomposition of $\mathrm{PBz}$, while only one peak could be observed in DTG curves of PBz composites. This tendency 
revealed that the introduction of fillers blocked the movement of molecules and reduced the crosslink density of the matrix, leading to the decrease in decomposition temperature of composites [42]. With further increases of filler contents, the degradation of PBz molecular chains was delayed because improved thermally conductivity of the composites led to the rapid spread of heat and ultrahigh specific heat capacity of $\mathrm{BN}$ resulted in the absorption of more heat.

(a)

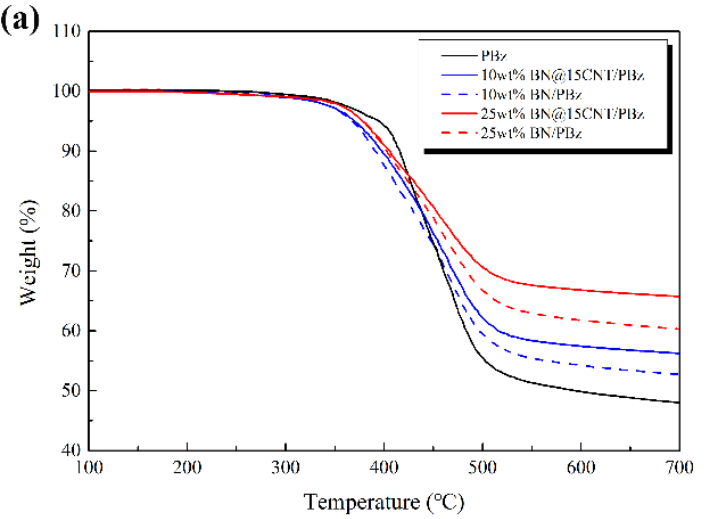

(b)

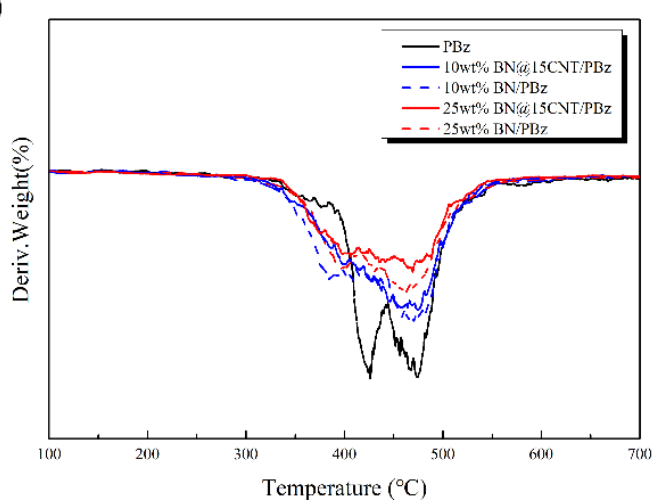

Figure 12. (a) TGA curves and (b) DTG curves of pristine $\mathrm{PBz}, \mathrm{BN} / \mathrm{PBz}$ composites and BN@15CNT/PBz composites.

Table 3. Corresponding characteristic thermal data of TGA curves

\begin{tabular}{cccc}
\hline \multirow{2}{*}{ Sample Name } & \multicolumn{2}{c}{ Weight Loss Temperature $\left({ }^{\circ} \mathbf{C}\right)$} & \multirow{2}{*}{$\mathbf{T}_{\text {heat-resistance index }}\left({ }^{\circ} \mathbf{C}\right)$} \\
\cline { 2 - 3 } & $\mathbf{T}_{\mathbf{5}}$ & $\mathbf{T}_{\mathbf{3 0}}$ & 212 \\
\hline PBZ & 394 & 460 & 208 \\
10 & 362 & 465 & 208 \\
wt\%BN@15CNT/PBZ & 367 & 462 & 218 \\
10 wt $\%$ BN/PBZ & 372 & 495 & 216 \\
25 & 378 & 483 & \\
wt $\%$ BN $15 \mathrm{CNT} / \mathrm{PBZ}$ & &
\end{tabular}

$\mathrm{T}_{\text {Heat-resistance index }}=0.49 \times\left(\mathrm{T} 5+0.6 \times\left(\mathrm{T}_{30}-\mathrm{T}_{5}\right)\right) \mathrm{T}_{5}$ and $\mathrm{T}_{30}$ is corresponding decomposition temperature of $5 \mathrm{wt} \%$ and $30 \mathrm{wt} \%$ weight loss, respectively.

In addition, the TG curves of BN@15CNT/PBz composites showed some interesting differences compared with that of BN/PBz composites while the DTG curves were similar. Slight decreases could be observed at the period of temperature between $150^{\circ} \mathrm{C}$ and $300^{\circ} \mathrm{C}$, due to the partial decomposition of a-CNT. Since BN did not decompose under $700^{\circ} \mathrm{C}$ while CNT did, the char yield of BN@15CNT/PBz composites should theoretically have been lower than that of $\mathrm{BN} / \mathrm{PBz}$ composites at the same filler loading, theoretically. However, the out-come was completely opposite. Not only was the residual mass of BN@15CNT/PBz composites higher than that of BN/PBz composites at the same filler loading, but also the margin between them increased with the addition of filler loading, which means the existence of CNTs could promote the formation of char residue [43]. In the meanwhile, the $\mathrm{T}_{\text {heat-resistance index }}\left({ }^{\circ} \mathrm{C}\right)$ of BN@15CNT/PBz and BN/PBz composites revealed that BN@15CNT hybrid fillers had more positive effect on the thermal stability of PBz composites than BN did, which became more obvious with the increase in filler loading. It can be inferred that the introduction of CNTs restricted the thermal motion of tether units, thereby minimizing the number of organic decomposition pathways accessible on the tether, resulting in the improvement of the composites' thermal properties.

\subsection{Mechanism of BN@CNT Hybrid Fillers Thermal Conductivity}

In general, it is believed that the high thermal boundary resistance $\left(R_{B}\right)$ between polymer matrix and fillers is the main factor that the experimental $\lambda$ values derive from theoretical predictions. A lot 
of theoretical models and simulation models of composites have been proposed in past decades to estimate the $R_{B}$ value. Herein, an effective medium approximation (EMA) was employed to analyze the obtained data in order to understand the effect of CNTs in the BN@15CNT hybrid filler on the improvement of the composites' thermal conductivity. The mathematical formula of $\lambda$ value of composites in the EMA model are shown as follows [13]:

$$
\begin{gathered}
\lambda_{\mathrm{c}}=\lambda_{\mathrm{m}} \frac{3+2 V_{\mathrm{f}}\left(\frac{\lambda_{\mathrm{f}}-\lambda_{\mathrm{m}}}{\lambda_{\mathrm{f}}}\right)}{3-V_{\mathrm{f}}\left(1-\alpha-\frac{\lambda_{\mathrm{m}}}{\lambda_{\mathrm{f}}}\right)} \\
\alpha=\frac{R_{B} \lambda_{\mathrm{m}}}{h}
\end{gathered}
$$

in which $\lambda_{\mathrm{f}}$ and $\lambda_{\mathrm{m}}$ stand for the thermal conductivity of the filler and matrix materials, respectively. $V_{f}$ is the volumetric fraction of filler. $R_{B}$ means thermal boundary resistance. $h$ represents thickness of the BN, which was estimated as $300 \mathrm{~nm}$ according to SEM observation. The thermally conductive coefficient of BN was $200 \mathrm{~W} \cdot \mathrm{m}^{-1} \cdot \mathrm{K}^{-1}$, and that of BN@15CNT can be estimated through the following equation [44]:

$$
\lambda=\lambda_{1} f+\lambda_{2}(1-f)
$$

in which $\lambda, \lambda_{1}$ and $\lambda_{2}$ represent the thermally conductive coefficient of the BN@15CNT hybrid filler, BN and CNTs, respectively. $f$ means the volumetric fraction of BN in the BN@15CNT hybrid filler. The $\lambda$ value of CNTs is $1300 \mathrm{~W} \cdot \mathrm{m}^{-1} \cdot \mathrm{K}^{-1}$. Therefore, the $\lambda$ value of the BN@15CNT hybrid filler is $290 \mathrm{~W} \cdot \mathrm{m}^{-1} \cdot \mathrm{K}^{-1}$.

Figure 13 and Table 4 show the fitted curves and parameters of the EMA model, with which the two $R_{B}$ values were calculated. The $R_{B}$ value of $B N / P B z$ was $2.194 \times 10^{-6} \mathrm{~m}^{2} \cdot \mathrm{K} \mathrm{W}^{-1}$, while that of BN@15CNT/PBz was $2.046 \times 10^{-6} \mathrm{~m}^{2} \cdot \mathrm{K} \mathrm{W}^{-1}$. The slight difference between them indicated the negligible effect of the CNTs on the heat conduction and thermal coupling to the PBz at low filler loading.

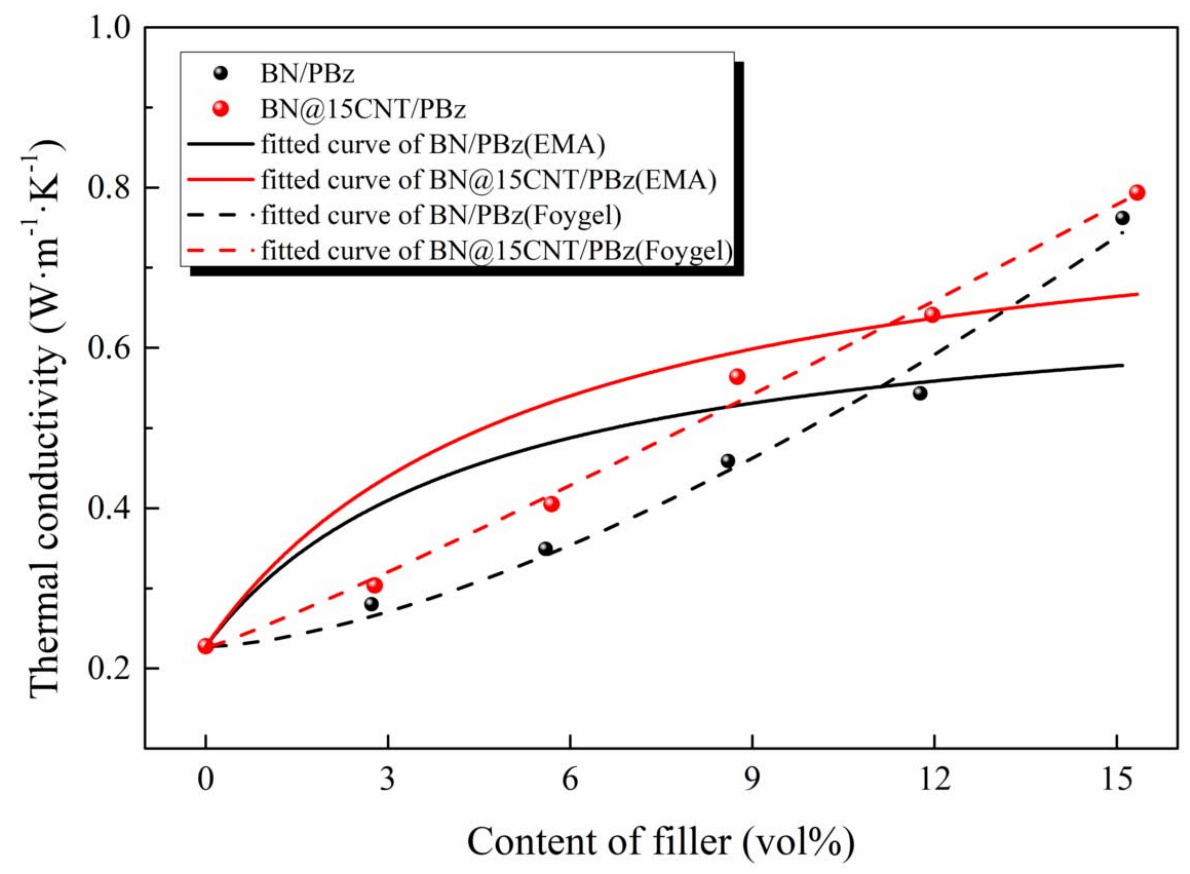

Figure 13. Fitted curves of $\lambda$ values of BN/PBz and BN@15CNT/PBz composites with the effective medium approximation (EMA) model and Foygel's model. 
Table 4. Fitted parameters of $\lambda$ values of BN/PBz and BN@15CNT/PBz composites with the EMA model and Foygel's model.

\begin{tabular}{ccccccc}
\hline & \multicolumn{2}{c}{ EMA } & \multicolumn{4}{c}{ Foygel } \\
\cline { 2 - 7 } & $\alpha$ & $\mathbf{R B}\left(\mathbf{m}^{\mathbf{2}} \cdot \mathbf{K} \cdot \mathbf{W}^{-\mathbf{1}}\right)$ & $\mathbf{V c}$ & $\boldsymbol{\beta}$ & $\mathbf{K}$ & $\mathbf{R c}\left(\mathbf{K}^{\left.-\mathbf{W}^{-1}\right)}\right.$ \\
\hline $\mathrm{BN} / \mathrm{PBz}$ & 1.665 & $2.194 \times 10^{-6}$ & 0.100 & 1.510 & 0.008 & $8.090 \times 10^{7}$ \\
$\mathrm{BN} @ 15 \mathrm{CNT} / \mathrm{PBz}$ & 1.553 & $2.046 \times 10^{-6}$ & 0.080 & 1.093 & 0.026 & $1.216 \times 10^{7}$ \\
\hline
\end{tabular}

The simple EMA model for BN and BN@15CNT is more accurate for the composites in which filler particles are separated by the polymer matrix. Therefore, the $\lambda$ values will be underestimated when the content of filler is sufficient for the construction of thermally conductive channels. With the increasing in filler loading, the main reason leading to phonon scattering turned into thermal contact resistance generated between filler instead of interfacial thermal resistance generated between filler and polymer matrix [45]. To illustrate the effect of BN@15CNT hybrid filler on enhancing thermal conductivity of PBz composites, the Foygel's model was employed to compute the contact thermal resistance $\left(R_{c}\right)$ between filler particles. The mathematical formulas of the $\lambda$ value of composites in Foygel's model are shown as follows [46]:

$$
\begin{gathered}
\lambda-\lambda_{\mathrm{m}}=\mathrm{K}\left(\frac{V_{\mathrm{f}}-V_{\mathrm{c}}}{1-V_{\mathrm{c}}}\right)^{\beta} \\
R_{\mathrm{c}}=\frac{1}{K d V_{\mathrm{c}}^{\beta}}
\end{gathered}
$$

in which $\lambda_{\mathrm{f}}$ and $\lambda_{\mathrm{m}}$ stand for the thermally conductive coefficient values of the filler and matrix materials, respectively. $K$ refers to a pre-exponential factor ratio which means the estimated contribution of individual filler networks, $\beta$ stands for a thermal conductivity exponent which reflects on the aspect ratio of the fillers, $V_{\mathrm{c}}$ refers to the critical volumetric fraction of fillers, and $d$ means diameter of fillers which is $50 \mu \mathrm{m}$ according to the supplier.

The fitted results and fitted curve for Foygel's model are shown in Figure 13 and Table 4 . The $R_{c}$ value for $\mathrm{BN} / \mathrm{PBz}$ was calculated to be $8.090 \times 10^{7} \mathrm{~K} \cdot \mathrm{W}^{-1}$, which was about seven times that of BN@15CNT/PBz, which was $1.216 \times 10^{7} \mathrm{~K} \cdot \mathrm{W}^{-1}$. The tremendous difference between them revealed that the phonon scatting phenomenon was undermined to a certain extent. The possible explanation was that CNTs served as interstitial material to connect adjacent BN platelets [47]. Therefore, the contact area was enlarged so that phonons could transfer throughout the composites with less resistance.

\section{Conclusions}

A novel hybrid filler (BN@CNT) with a hierarchical "line-plane" structure was successfully fabricated through a condensation reaction. A series of hybrid fillers prepared with different mass ratios between BN and CNTs, ranging from 100:5 to 100:20. It has been proven that BN@15CNT hybrid fillers exhibited the best abilities in promoting thermal conductivity of PBz composites compared to the other ones, because with insufficient content of CNTs, the edge of BN platelets cannot be covered with CNTs, which hindered the connection between hybrid fillers. The $\lambda$ value of PBz composite reached $0.794 \mathrm{~W} \cdot \mathrm{m}^{-1} \cdot \mathrm{K}^{-1}$ with $25 \mathrm{wt} \% \mathrm{BN} @ 15 \mathrm{CNT}$ hybrid filler, which can be further improved to $0.865 \mathrm{~W} \cdot \mathrm{m}^{-1} \cdot \mathrm{K}^{-1}$ with $15 \mathrm{wt} \%$ of BN@15CNT and $10 \mathrm{wt} \%$ of BN. Theoretical fitting revealed that CNTs acted as an interstitial material, transferring phonons between BN platelets. The $\varepsilon$ and $\tan \delta$ values of $\mathrm{PBz}$ composites increases slightly with the increasing content of BN@15CNT hybrid filler. Moreover, the prepared $\mathrm{PBz}$ composites maintain high electrically insulating properties, showing their potential applications in packaging materials and circuit boards. TGA revealed that the BN@15CNT hybrid filler slightly increase thermal stability of the composites and promoted the formation of char residue. 
Author Contributions: Conceptualization, Y.W.; methodology, Y.W.; software, F.T.; validation, Y.W.; formal analysis, Z.H.; investigation, K.S.; resources, D.D.; data curation, C.L.; writing一 original draft preparation, Y.W.; writing-review and editing, Y.W.; visualization, Y.W.; supervision, W.W. and D.D.; project administration, W.W.; funding acquisition, W.W. All authors have read and agreed to the published version of the manuscript.

Funding: The work was funded by the Chinese German Centre for the Promotion of Science via the National Natural Science Foundation of China (NSFC) and Deutsche Forschungsgemeinschaft (DFG) under grant number GZ1448.

Conflicts of Interest: The authors declare no conflict of interest.

\section{References}

1. McGlen, R.J.; Jachuck, R.; Lin, S. Integrated thermal management techniques for high power electronic devices. Appl. Thermal Eng. 2004, 24, 1143-1156. [CrossRef]

2. Guo, Y.; Ruan, K.; Yang, X.; Ma, T.; Kong, J.; Wu, N.; Zhang, J.; Gu, J.; Guo, Z. Constructing fully carbon-based fillers with a hierarchical structure to fabricate highly thermally conductive polyimide nanocomposites. J. Mater. Chem. C 2019, 7, 7035-7044. [CrossRef]

3. Khan, J.; Momin, S.A.; Mariatti, M. A review on advanced carbon-based thermal interface materials for electronic devices. Carbon 2020, 168, 65-112. [CrossRef]

4. Shen, H.; Guo, J.; Wang, H.; Zhao, N.; Xu, J. Bioinspired Modification of h-BN for High Thermal Conductive Composite Films with Aligned Structure. ACS Appl. Mater. Interfaces 2015, 7, 5701-5708. [CrossRef] [PubMed]

5. Kelly, A.G.; Hallam, T.; Backes, C.; Harvey, A.; Esmaeily, A.S.; Godwin, I.; Coelho, J.; Nicolosi, V.; Lauth, J.; Kulkarni, A.; et al. All-printed thin-film transistors from networks of liquid-exfoliated nanosheets. Science 2017, 356, 69-73. [CrossRef]

6. Guo, Y.; Yang, X.; Ruan, K.; Kong, J.; Dong, M.; Zhang, J.; Gu, J.; Guo, Z. Reduced Graphene Oxide Heterostructured Silver Nanoparticles Significantly Enhanced Thermal Conductivities in Hot-Pressed Electrospun Polyimide Nanocomposites. ACS Appl. Mater. Interfaces 2019. [CrossRef]

7. Lee, S.H.; Kim, K.S.; Shim, J.H.; Ahn, C.-H. High-Performance Printed Circuit Board Materials Based on Benzoxazine and Epoxy Blend System. Macromol. Res. 2018, 26, 388-393. [CrossRef]

8. Kiskan, B.; Aydogan, B.; Yagci, Y. Synthesis, characterization, and thermally activated curing of oligosiloxanes containing benzoxazine moieties in the main chain. J. Polym. Sci. A Polym. Chem. 2009, 47, 804-811. [CrossRef]

9. Ning, X.; Ishida, H. Phenolic materials via ring-opening polymerization: Synthesis and characterization of bisphenol-A based benzoxazines and their polymers. J. Polym. Sci. Part A Polym. Chem. 1994, 32, 1121-1129. [CrossRef]

10. Takeichi, T.; Saito, Y.; Agag, T.; Muto, H.; Kawauchi, T. High-performance polymer alloys of polybenzoxazine and bismaleimide. Polymer 2008, 49, 1173-1179. [CrossRef]

11. Liu, Y.; Chen, J.; Zhang, Y.; Gao, S.; Lu, Z.; Xue, Q. Highly thermal conductive benzoxazine-epoxy interpenetrating polymer networks containing liquid crystalline structures. J. Polym. Sci. Part B Polym. Phys. 2017, 55, 1813-1821. [CrossRef]

12. Yu, A.; Ramesh, P.; Sun, X.; Bekyarova, E.; Itkis, M.E.; Haddon, R.C. Enhanced Thermal Conductivity in a Hybrid Graphite Nanoplatelet - Carbon Nanotube Filler for Epoxy Composites. Adv. Mater. 2008, 20, 4740-4744. [CrossRef]

13. Burger, N.; Laachachi, A.; Ferriol, M.; Lutz, M.; Toniazzo, V.; Ruch, D. Review of thermal conductivity in composites: Mechanisms, parameters and theory. Prog. Polym. Sci. 2016, 61, 1-28. [CrossRef]

14. Zare, Y.; Shabani, I. Polymer/metal nanocomposites for biomedical applications. Mater. Sci. Eng. C 2016, 60, 195-203. [CrossRef] [PubMed]

15. Im, H.; Kim, J. Thermal conductivity of a graphene oxide-carbon nanotube hybrid/epoxy composite. Carbon 2012, 50, 5429-5440. [CrossRef]

16. Chang, C.W.; Fennimore, A.M.; Afanasiev, A.; Okawa, D.; Ikuno, T.; Garcia, H.; Li, D.; Majumdar, A.; Zettl, A. Isotope Effect on the Thermal Conductivity of Boron Nitride Nanotubes. Phys. Rev. Lett. 2006, 97, 085901. [CrossRef] 
17. Shen, Z.; Bateman, S.; Wu, D.; Mcmahon, P.; Dellolio, M.; Gotama, J. The effects of carbon nanotubes on mechanical and thermal properties of woven glass fibre reinforced polyamide- 6 nanocomposites. Compos. Sci. Technol. 2009, 69, 239-244. [CrossRef]

18. Wang, F.; Zhang, K.; Liang, W.; Wang, Z.; Yang, B. Experimental and analytical studies on the flexible, low-voltage electrothermal film based on the multi-walled carbon nanotube/polymer nanocomposite. Nanotechnology 2019, 30, 065704. [CrossRef]

19. Das, A.; Stöckelhuber, K.W.; Jurk, R.; Saphiannikova, M.; Fritzsche, J.; Lorenz, H.; Klüppel, M.; Heinrich, G. Modified and unmodified multiwalled carbon nanotubes in high performance solution-styrene-butadiene and butadiene rubber blends. Polymer 2008, 49, 5276-5283. [CrossRef]

20. Lule, Z.; Kim, J. Surface Modification of Aluminum Nitride to Fabricate Thermally Conductive poly(Butylene Succinate) Nanocomposite. Polymers 2019, 11, 148. [CrossRef]

21. Yu, C.; Zhang, J.; Li, Z.; Tian, W.; Wang, L.; Luo, J.; Li, Q.; Fan, X.; Yao, Y. Enhanced through-plane thermal conductivity of boron nitride/epoxy composites. Compos. Part A Appl. Sci. Manuf. 2017, 98, 25-31. [CrossRef]

22. Yang, Y.; Gao, J.; Lei, T.; Yang, J.; Wang, J.; Liu, J. Thermal conductivity and mechanical properties of polyimide composites with mixed fillers of $\mathrm{BN}$ flakes and $\mathrm{SiC} @ \mathrm{SiO}_{2}$ whiskers. Polym. Eng. Sci. 2020, 60, 1044-1053. [CrossRef]

23. Su, Z.; Wang, H.; Ye, X.; Tian, K.; Huang, W.; He, J.; Guo, Y.; Tian, X. Anisotropic Thermally Conductive Flexible Polymer Composites Filled with Hexagonal Born Nitride (h-BN) Platelets and Ammine Carbon Nanotubes (CNT-NH2): Effects of the Filler Distribution and Orientation. Compos. Part A Appl. Sci. Manuf. 2018, 109, 402-412. [CrossRef]

24. Lei, Y.; Han, Z.; Ren, D.; Pan, H.; Xu, M.; Liu, X. Design of h-BN-Filled Cyanate/Epoxy Thermal Conductive Composite with Stable Dielectric Properties. Macromol. Res. 2018, 26, 602-608. [CrossRef]

25. Agari, Y.; Ueda, A.; Nagai, S. Thermal conductivities of composites in several types of dispersion systems. J. Appl. Polym. Sci. 1991, 42, 1665-1669. [CrossRef]

26. Balandin, A.A. Thermal properties of graphene and nanostructured carbon materials. Nat. Mater. 2011, 10, 569-581. [CrossRef]

27. Xue, Y.; Li, X.; Wang, H.; Zhang, D.; Chen, Y. Thermal conductivity improvement in electrically insulating silicone rubber composites by the construction of hybrid three-dimensional filler networks with boron nitride and carbon nanotubes. J. Appl. Polym. Sci. 2019, 136, 46929. [CrossRef]

28. Grady, B.P. Recent Developments Concerning the Dispersion of Carbon Nanotubes in Polymers. Macromol. Rapid Commun. 2010, 31, 247-257. [CrossRef]

29. Gong, X.; Liu, Y.; Wang, Y.; Xie, Z.; Dong, Q.; Dong, M.; Liu, H.; Shao, Q.; Lu, N.; Murugadoss, V.; et al. Amino graphene oxide/dopamine modified aramid fibers: Preparation, epoxy nanocomposites and property analysis. Polymer 2019, 168, 131-137. [CrossRef]

30. Yao, Y.; Sun, J.; Zeng, X.; Sun, R.; Xu, J.-B.; Wong, C.-P. Construction of 3D Skeleton for Polymer Composites Achieving a High Thermal Conductivity. Small 2018. [CrossRef]

31. Zhang, S.; Tian, Y.; Gu, X.; Tang, W.; Sun, J. Improving the flame resistance and thermal conductivity of ethylene-vinyl acetate composites by incorporating hexachlorocyclotriphosphazene-modified graphite and carbon nanotubes. Polym. Compos. 2018, 39, E891-E901. [CrossRef]

32. Zhang, J.; Yin, W.; Shang, H.; Liu, C. In situ FT-IR spectroscopy investigations of carbon nanotubes supported Co-Mo catalysts for selective hydrodesulfurization of FCC gasoline. J. Nat. Gas Chem. 2008, 17, 165-170. [CrossRef]

33. Kim, K.; Kim, M.; Kim, J. Thermal and mechanical properties of epoxy composites with a binary particle filler system consisting of aggregated and whisker type boron nitride particles. Compos. Sci. Technol. 2014, 103, 72-77. [CrossRef]

34. Yang, J.; Qi, G.-Q.; Tang, L.-S.; Bao, R.-Y.; Bai, L.; Liu, Z.-Y.; Yang, W.; Xie, B.-H.; Yang, M.-B. Novel photodriven composite phase change materials with bioinspired modification of BN for solar-thermal energy conversion and storage. J. Mater. Chem. A 2016, 4, 9625-9634. [CrossRef]

35. Wang, Y.; Wu, W.; Drummer, D.; Liu, C.; Shen, W.; Tomiak, F.; Schneider, K.; Liu, X.; Chen, Q. Highly thermally conductive polybenzoxazine composites based on boron nitride flakes deposited with copper particles. Mater. Design 2020, 191, 108698. [CrossRef] 
36. Liang, W.; Wang, F.; Tay, T.E.; Yang, B.; Wang, Z. Experimental and Analytical Investigation of Epoxy/MWCNT Nanocomposites: Electrical, Thermal Properties, and Electric Heating Behavior. Polym. Eng. Sci. 2020, 60, 233-242. [CrossRef]

37. Kaleemullah, M.; Khan, S.U.; Kim, J.-K. Effect of surfactant treatment on thermal stability and mechanical properties of CNT/polybenzoxazine nanocomposites. Compos. Sci. Technol. 2012, 72, 1968-1976. [CrossRef]

38. Xu, M.; Lei, Y.; Ren, D.; Chen, S.; Chen, L.; Liu, X. Synergistic Effects of Functional CNTs and h-BN on Enhanced Thermal Conductivity of Epoxy/Cyanate Matrix Composites. NANOMATERIALS 2018, 8, 997. [CrossRef] [PubMed]

39. Ayesh, A.S. Dielectric Relaxation and Thermal Stability of Polycarbonate Doped with $\mathrm{MnCl}_{2}$ Salt. J. Therm. Compos. Mater. 2008, 21, 309-322. [CrossRef]

40. Zeng, M.; Wang, J.; Li, R.; Liu, J.; Chen, W.; Xu, Q.; Gu, Y. The curing behavior and thermal property of graphene oxide/benzoxazine nanocomposites. Polymer 2013, 54, 3107-3116. [CrossRef]

41. Farrag, E.A.M. Dielectric relaxation behavior of three-phase MWCNTs-PANI polystyrene nanocomposites. J. Therm. Compos. Mater. 2019, 32, 884-894. [CrossRef]

42. Ren, J.; Li, Q.; Yan, L.; Jia, L.; Huang, X.; Zhao, L.; Ran, Q.; Fu, M. Enhanced thermal conductivity of epoxy composites by introducing graphene@boron nitride nanosheets hybrid nanoparticles. Mater. Design 2020, 191, 108663. [CrossRef]

43. Zhang, X.; Wu, K.; Liu, Y.; Yu, B.; Zhang, Q.; Chen, F.; Fu, Q. Preparation of highly thermally conductive but electrically insulating composites by constructing a segregated double network in polymer composites. Ccompos. Sci. Technol. 2019, 175, 135-142. [CrossRef]

44. Wang, F.; Yao, Y.; Zeng, X.; Huang, T.; Sun, R.; Xu, J.; Wong, C.-P. Highly thermally conductive polymer nanocomposites based on boron nitride nanosheets decorated with silver nanoparticles. RSC Adv. 2016, 6, 41630-41636. [CrossRef]

45. Kim, H.S.; Kim, J.H.; Kim, W.Y.; Lee, H.S.; Kim, S.Y.; Khil, M.-S. Volume control of expanded graphite based on inductively coupled plasma and enhanced thermal conductivity of epoxy composite by formation of the filler network. Carbon 2017, 119, 40-46. [CrossRef]

46. Foygel, M.; Morris, R.D.; Anez, D.; French, S.; Sobolev, V.L. Theoretical and computational studies of carbon nanotube composites and suspensions: Electrical and thermal conductivity. Phys. Rev. B 2005, 71, 104201. [CrossRef]

47. Shahil, K.M.F.; Balandin, A.A. Thermal properties of graphene and multilayer graphene: Applications in thermal interface materials. Solid State Commun. 2012, 152, 1331-1340. [CrossRef] 